ITC $4 / 48$

Information Technology and Control

Vol. 48 / No. 4/ 2019

pp. 660-672

DOI 10.5755/j01.itc.48.4.24297
Robust Backstepping Sliding Mode Control with $L_{2}$-Gain Performance for Reference Input Wheel Slip Tracking of Vehicle

\title{
Robust Backstepping Sliding Mode Control with $L_{2}$-Gain Performance for Reference Input Wheel Slip Tracking of Vehicle
}

\section{Jiaxu Zhang}

State key Laboratory of Automotive Simulation and Control, Jilin University, Changchun 130022, China;

Research and Development Center, China FAW Group Corporation, Changchun 130022, China;

e-mail:zhjx_686@163.com

\section{Jing Li}

State key Laboratory of Automotive Simulation and Control, Jilin University, Changchun 130022, China; e-mail:liye1129@163.com

Corresponding author: liye1129@163.com

The wheel slip control is the basis of active safety control systems and intelligent driver assistance systems. This paper presents a new robust backstepping sliding mode controller for reference input wheel slip tracking based on the single-corner model combined with the actuator dynamics. The proposed controller is realized by combining backstepping method, which has the merits of simplified and flexible design procedure, with sliding mode control, which has robustness against system uncertainty and external disturbance. Moreover, the closed-loop wheel dynamic system is $L_{2}$-gain stable by Lyapunov-based method, and the simulation results show that the proposed controller has better performance.

KEYWORDS:Wheel slip control, Backstepping method, Sliding mode control, $L_{2}$-gain stable, Robustness.

\section{Introduction}

Active safety control systems and intelligent driver assistance systems can effectively enhance driving safety and maneuvering stability, and greatly lighten the physical and psychological burden of the driver, 
especially in traffic jams or long-distance driving circumstances. However, the wheel slip control is the basis of active safety control systems and intelligent driver assistance systems. For instance, the anti-lock brake system (ABS) regulates the slip of each wheel at its optimum value to prevent it from locking during braking, such that the shortest stopping distance is achieved and the capability of directional stability and steer-handling is maintained [4]. The electronic stability program (ESP) may produce additional yaw moment by commanding the target slip of one or two wheels to prevent vehicle from spinning and drifting out of lane [29]. Finally, the adaptive cruise control system (ACC) can follow target speed or forward vehicle at the desired safety headway distance by commanding the target slip of the wheels and the target torque of the power system [16]. As a consequence, designing the wheel slip controller has important theoretical and practical significance for active safety control systems and intelligent driver assistance systems.

In recent years, many control approaches which are robust against system uncertainty and external disturbance have been proposed for the wheel slip control due to the modeling errors, the measurement or estimation errors, and the changing of external environment conditions of the wheel dynamic system, such as sliding mode control [23], hybrid control [25] and fuzzy control [13], etc. Johansen et al. [9] established the speed-dependent nominal linearized slip model with a perturbation term as a basis for the wheel slip control, and utilized gain-scheduled LQR approach to design the gain matrices of the controller. Pasillas-Lépine [19] adopted wheel deceleration logic-based switching and wheel dynamic model to design the five-phase anti-lock brake algorithm, and proved the existence and stability of limit cycles by the Poincaré map. Hsu [7] proposed an intelligent exponential sliding-mode control strategy for ABS, and a functional recurrent fuzzy neural network uncertainty estimator was designed to reduce the chattering of the exponential sliding-mode control strategy by approximating and compensating the unknown nolinear term of ABS dynamics on-line. Jing et al. [8] presented a switched control strategy for the anti-lock brake system and then analyzed the stability condition of the closed-loop system by Lyapunov-based method in the Filippov framework. The proposed control strategy in $[7-9,19]$ may only regulate the wheel slip at its optimum point to generate the maximum braking force. However, the continuous wheel slip tracking control is the basis of active safety control systems and intelligent driver assistance systems. Tanelli et al. [24] proposed a nonlinear output feedback controller with input constraints for active braking control systems by Lyapunov-based method. The proposed algorithm could produce the limit cycles when the desired slip is in the unstable region of the friction curve, thereby enhance braking safety and performance by detecting the existence of limit cycles. Mirzaei and Mirzaeinejad [14] adopted an optimal predictive approach to design a nonlinear wheel slip controller based on a single-corner vehicle model, and proved the proposed approach was robust against system uncertainties via Lyapunov-based method. Park and Lim [18] established the quarter car model with the time delay on the brake torque as nominal model and adopted adaptive sliding mode control with the reduction method and the nonlinear vehicle speed observer to design the output feedback controller of the wheel slip, which could drive the wheel slip to track the desired value. Harifi et al. [6] utilized the double-corner vehicle model with the Burckhardt tire friction model as nominal model and proposed a sliding mode control with integral switching surface to design the robust wheel slip controllers for front and rear wheels separately. In $[6,18]$, the traditional sliding mode control can effectively suppress the uncertainty of the system, but cause the chattering of the system. Amodeo et al. [2] proposed a novel wheel slip controller based on second-order sliding-mode approach, which has higher accuracy and robustness feature with respect to parameter uncertainties and disturbances and then adopted first-order sliding-mode observer to estimate the peak tire-road friction coefficient. Lin and Hsu [12] proposed a hybrid control system composed of an ideal controller and a compensation controller for the anti-lock brake system. The ideal controller with a neural network observer which was used to estimate the system uncertainties was designed to maintain the wheel slip at the desired slip, and the compensation controller was designed to have robustness against the approximation error of the neural network observer. Mirzaeinejad [15] presented a new robust prediction-based nonlinear wheel slip controller in 
conjunction with radial basis function neural network (RBFNN), which was used to improve the robustness of the system by estimating the unknown uncertainties of the system on-line. Sardarmehni et al. [20] proposed two model-free wheel slip controller on the basis of fuzzy logic control approach and neural predictive control aproach, and simulation results showed that the neural predictive control approach had more robustness against exogenous disturbances and modeling uncertainties. In [2, 12, $15,20]$, the uncertainty observer improves the robustness of the system, but increases the complexity of the system. Therefore, it is absolutely essential to design the wheel slip controller which has both robustness against system uncertainty and external disturbance and simple system structure.

The standard backstepping method introduced in $[5,17]$ provides a recursive Lyapunov-based framework for the controller design of the lower-triangular nonlinear system and has the merits of simplified and flexible design procedure. However, the standard backstepping method is not robust against the system uncertainty and external disturbance. This paper surmounts the demerit of the standard backstepping method by combining the standard backstepping method with sliding mode control which is insensitive to system uncertainty and external disturbance, and a backstepping sliding mode design framework is proposed. Compared with the nonlinear $L_{2}$-gain control method [28], the backstepping sliding mode design framework can avoid solving the complex Hamilton-Jacobi-Issacs inequality to attain the same control objective that the ratio of the $L_{2}$ norm of the system output to the $L_{2}$ norm of the lumped disturbance is less than the given threshold value. Then, a new robust backstepping sliding mode controller (RBSMC) with $L_{2}$-gain performance for reference input wheel slip tracking is derived based on a single-corner vehicle model with actuator dynamics and the backstepping sliding mode design framework. Moreover, the effectiveness of the proposed controller is verified based on vehicle dynamics simulation software.

This paper is organized as follows. Section 2 provides the dynamic model. Section 3 shows the nonlinear robust controller. Section 4 introduces our simulations and results. Finally, Section 5 draws the main conclusion of our work.

\section{System Modelling}

The proposed RBSMC for reference input wheel slip tracking is based on a single-corner model, as it provides a simple yet sufficiently rich description of the braking dynamics. As shown in Figure 1, the degrees of freedom for the model consists of the vehicle speed and the angular speed of the wheel.

\section{Figure 1}

The single-corner model

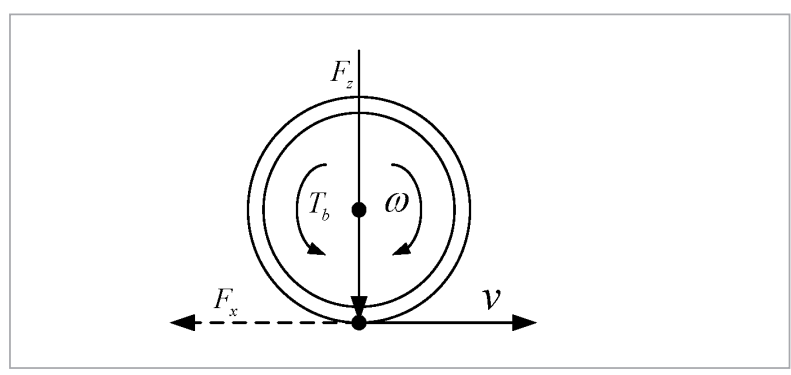

In order to simplify the nonlinear controller design, the following modeling assumptions are made:

1 the vehicle is moving on a flat horizontal plane;

2 the suspension dynamics are neglected;

3 the wheel radius is assumed to be constant;

4 the tire camber and the tire sideslip angle are assumed to be zero;

5 the tire relaxation dynamics are neglected.

The single-corner model is given by [21]

$J \dot{\omega}=r F_{x}-T_{b}$
$m \dot{v}=-F_{x}$,

where $\omega$ is the angular speed of the wheel; $v$ is the vehicle speed; $T_{b}$ is the braking torque; $F_{x}$ is the longitudinal tire-road contact force; $J, m$ and $r$ are the wheel inertia, the single-corner mass and the effective rolling wheel radius, respectively.

The wheel slip $\lambda$ is defined by

$$
\lambda=\frac{v-\omega r}{v} .
$$

Notice that, during braking, as $\omega r \leq v, \lambda \in[0,1]$. In this paper, the tire model introduced by Burckhardt 
[3] has been used to describe the nonlinear relationship of the tire-road friction coefficient and the wheel slip, as it is simple and has a good degree of accuracy:

$$
\mu(\lambda)=\vartheta_{r 1}\left(1-e^{-\lambda \vartheta_{r 2}}\right)-\lambda \vartheta_{r 3},
$$

where $\vartheta_{r 1}$ is the maximum value of friction curve; $\vartheta_{r 2}$ is the friction curve shape; $\vartheta_{r 3}$ is the friction curve difference between the maximum value and the value at $\lambda=1$. By changing the values of these three parameters, many different tire-road friction conditions can be modeled. The parameters of Burckhardt model for different road surfaces are listed in Table 1 [10].

\section{Table 1}

Parameters of Burckhardt model for different road surfaces

\begin{tabular}{l|c|c|c}
\hline \multicolumn{1}{|c|}{ Surface conditions } & $\boldsymbol{\vartheta}_{r 1}$ & $\boldsymbol{\vartheta}_{r 2}$ & $\boldsymbol{\vartheta}_{r 3}$ \\
\hline Dry asphalt & 1.2801 & 23.99 & 0.52 \\
\hline Wet asphalt & 0.857 & 33.822 & 0.347 \\
\hline Dry concrete & 1.1973 & 25.168 & 0.5373 \\
\hline Dry cobblestones & 1.3713 & 6.4565 & 0.6691 \\
\hline Wet cobblestones & 0.4004 & 33.708 & 0.1204 \\
\hline Snow & 0.1946 & 94.129 & 0.0646 \\
\hline Ice & 0.05 & 306.39 & 0 \\
\hline
\end{tabular}

The longitudinal tire-road contact force is expressed as

$$
F_{x}=F_{z} \mu(\lambda)
$$

where $F_{z}$ is the vertical force at the tire-road contact point.

The derivative of Eq. (3) with respect to time yields

$$
\dot{\lambda}=\frac{1}{v}[(1-\lambda) \dot{v}-\dot{\omega} r]
$$

Substituting Eqs. (1), (2) and (5) into Eq. (6) yields

$$
\dot{\lambda}=-\frac{1}{v}\left(\frac{1-\lambda}{m}+\frac{r^{2}}{J}\right) F_{z} \mu(\lambda)+\frac{r}{J v} T_{b} .
$$

Time delays may cause instability and performance deterioration of system [30]. Taking brake lag into account, the actuator dynamics [27] is given by

$$
T_{b}=\frac{1}{\tau_{b} s+1} u,
$$

where $\tau_{b}$ is dimensionless time constant; $u$ is the actual control input.

The derivate of Eq. (8) with respect to time yields

$$
\dot{T}_{b}=-\frac{1}{\tau_{b}} T_{b}+\frac{1}{\tau_{b}} u .
$$

Since the vehicle speed dynamics are much slower than the wheel slip dynamics due to large differences in inertia, the vehicle speed $v_{\text {can }}$ be regarded as a slowly-varying parameter. Hence, the Eq. (2) can be neglected, and we consider only the wheel slip dynamics with the actuator dynamics. Furthermore, we define the state variables $x_{1}=\lambda-\lambda_{d}, x_{2}=T_{b}$, where $\lambda_{d}$ is the reference input wheel slip. Eqs.(7) and (9) are merged together into the state-space form of the nominal model as follows

$$
\left\{\begin{array}{l}
\dot{x}_{1}=-\frac{1}{v}\left(\frac{1-x_{1}-\lambda_{d}}{m}+\frac{r^{2}}{J}\right) F_{z} \mu\left(x_{1}+\lambda_{d}\right)+\frac{r}{J v} x_{2} \\
\dot{x}_{2}=-\frac{1}{\tau_{b}}\left(x_{2}-u\right)
\end{array} .\right.
$$

Consider the nominal model with the lumped uncertainty, Eq.(10) can be rewritten as

$$
\psi_{1}:\left\{\begin{array}{l}
\dot{x}_{1}=f\left(x_{1}\right)+G x_{2}+d_{1} \\
\dot{x}_{2}=-\frac{1}{\tau_{b}}\left(x_{2}-u\right)+d_{2}
\end{array},\right.
$$

where

$f\left(x_{1}\right)=-\frac{1}{v}\left(\frac{1-x_{1}-\lambda_{d}}{m}+\frac{r^{2}}{J}\right) F_{z} \mu\left(x_{1}+\lambda_{d}\right), G=\frac{r}{J v}$ and $d=\left[\begin{array}{ll}d_{1} & d_{2}\end{array}\right]^{\mathrm{T}}$ is the lumped uncertainty that contains system uncertainty and external disturbance.

\section{Robust Backstepping Sliding Mode Controller Design}

In this section, sliding mode control combined with backstepping method is used to derive the RBSMC for reference input wheel slip tracking, which integrates 
both the merits of sliding mode control and backstepping method. Based on the standard backstepping method, we employ the change of coordinates

$$
\left\{\begin{array}{l}
z_{1}=x_{1} \\
z_{2}=x_{2}-\alpha_{1}
\end{array},\right.
$$

where $\alpha_{1}$ is virtual controller.

Define the output of the system $\psi_{1} \quad z=\left[\begin{array}{ll}\kappa_{1} z_{1} & \kappa_{2} z_{2}\end{array}\right]^{\mathrm{T}}$, where $\kappa$, and $\kappa_{\text {}}$ are nonnegative weight coefficients, and the system $\psi_{1}$ is rewritten

$$
\psi_{2}:\left\{\begin{array}{l}
\dot{x}_{1}=f\left(x_{1}\right)+G x_{2}+d_{1} \\
\dot{x}_{2}=-\frac{1}{\tau_{b}}\left(x_{2}-u\right)+d_{2} \\
z=\left[\begin{array}{ll}
\kappa_{1} z_{1} & \kappa_{2} z_{2}
\end{array}\right]^{\mathrm{T}}
\end{array} .\right.
$$

The aim of this paper is to design a RBSMC for the system $\psi_{2}$ such that the closed-loop wheel dynamic system is asymptotically stable when the disturbance $d=0$, and is $\mathrm{L}_{2}$-gain stable when the disturbance $d \neq 0$, which means that the relationship of the output of the system $\psi_{2}$ and the disturbance satisfies the following inequality

$$
\int_{0}^{\mathrm{T}}\|z\|^{2} d t \leq \gamma^{2} \int_{0}^{\mathrm{T}}\|d\|^{2} d t
$$

for all $T \geq 0$ and all $d \in L_{2}(0, T)$, where $\gamma$ is positive constant.

The design procedure is elaborated in the following steps.

Step 1: Define the first storage function $V_{1}=\frac{1}{2} z_{1}^{2}$. Note that

$$
\dot{z}_{1}=\dot{x}_{1}=f\left(x_{1}\right)+G x_{2}+d_{1}=f\left(x_{1}\right)+G\left(z_{2}+\alpha_{1}\right)+d_{1} .
$$

Differentiating the first storage function $V_{1}$ along the trajectories of the system $\psi_{2}$ and substituting Eq. (15) into it, it is easy to have

$$
\dot{V}_{1}=z_{1} \dot{z}_{1}=z_{1}\left(f\left(x_{1}\right)+G\left(z_{2}+\alpha_{1}\right)+d_{1}\right) .
$$

By viewing $x_{2}$ as a virtual control input, let us choose virtual controller $\alpha_{1}$ as follows
$\alpha_{1}=-G^{-1}\left(c_{1} z_{1}+f\left(x_{1}\right)\right)$,

where $c_{1}$ is positive constant, and then

$\dot{V}_{1}=z_{1}\left(-c_{1} z_{1}+G z_{2}+d_{1}\right)$.

Define the function

$$
\begin{aligned}
H_{1}= & \dot{V}_{1}+\frac{1}{2}\left(\|z\|^{2}-\gamma^{2}\left\|d_{1}\right\|^{2}\right) \\
= & z_{1}\left(-c_{1} z_{1}+G z_{2}+d_{1}\right) \\
& +\frac{1}{2}\left(\kappa_{1}^{2} z_{1}^{2}+\kappa_{2}^{2} z_{2}^{2}-\gamma^{2} d_{1}^{2}\right)
\end{aligned} .
$$

Step 2: Define the sliding surface $\sigma=c_{0} z_{1}+z_{2}$, where $c_{0}$ is the design parameter. Note that

$$
\dot{z}_{2}=\dot{x}_{2}-\dot{\alpha}_{1}=-\frac{1}{\tau_{b}}\left(x_{2}-u\right)+d_{2}-\dot{\alpha}_{1},
$$

where

$$
\begin{gathered}
\dot{\alpha}_{1}=-G^{-1}\left(c_{1} \dot{z}_{1}+\frac{\partial f\left(x_{1}\right)}{\partial x_{1}} \dot{x}_{1}\right) \\
=-G^{-1}\left(c_{1}+\frac{\partial f\left(x_{1}\right)}{\partial x_{1}}\right)\left(-c_{1} z_{1}+G z_{2}+d_{1}\right) \\
\frac{\partial f\left(x_{1}\right)}{\partial x_{1}}=-\frac{1}{v}\left(\left(\frac{1-x_{1}-\lambda_{d}}{m}+\frac{r^{2}}{J}\right) F_{z} \frac{\partial \mu\left(x_{1}+\lambda_{d}\right)}{\partial x_{1}}\right) .
\end{gathered}
$$

Differentiating the sliding surface $\sigma$ and substituting Eqs. (15) and (20) into it, it is easy to have

$$
\begin{gathered}
\dot{\sigma}=c_{0} \dot{z}_{1}+\dot{z}_{2}=c_{0}\left(-c_{1} z_{1}+G z_{2}+d_{1}\right) \\
-\frac{1}{\tau_{b}}\left(x_{2}-u\right)+d_{2}-\dot{\alpha}_{1} .
\end{gathered}
$$

Augment the storage function of Step 1, and thus the new storage function is given by

$V_{2}=V_{1}+\frac{1}{2} \sigma^{2}$.

Define the function 


$$
H_{2}=\dot{V}_{2}+\frac{1}{2}\left(\|z\|^{2}-\gamma^{2}\|d\|^{2}\right) .
$$

Substituting Eq. (19) into Eq. (25) yields

$$
\begin{aligned}
H_{2} & =\dot{V}_{1}+\sigma \dot{\sigma}+\frac{1}{2}\left(\|z\|^{2}-\gamma^{2}\|d\|^{2}\right)=H_{1}+\sigma \dot{\sigma}-\frac{1}{2} \\
& =z_{1}\left(-c_{1} z_{1}+G z_{2}+d_{1}\right)+\frac{1}{2}\left(\kappa_{1}^{2} z_{1}^{2}+\kappa_{2}^{2} z_{2}^{2}-\gamma^{2},\right. \\
& +\sigma \dot{\sigma}-\frac{1}{2} \gamma^{2} d_{2}^{2}=z_{1}\left(-c_{1} z_{1}+G z_{2}+d_{1}\right) \\
& +\frac{1}{2}\left(\kappa_{1}^{2} z_{1}^{2}+\kappa_{2}^{2} z_{2}^{2}-\gamma^{2} d_{1}^{2}-\gamma^{2} d_{2}^{2}\right) \\
& +\sigma\left(\begin{array}{l}
\left.c_{0}\left(-c_{1} z_{1}+G z_{2}+d_{1}\right)-\frac{1}{\tau_{b}}\left(x_{2}-u\right)+d_{2}\right) \\
\left.+G^{-1}\left(c_{1}+\frac{\partial f\left(x_{1}\right)}{\partial x_{1}}\right)\left(-c_{1} z_{1}+G z_{2}+d_{1}\right)\right)
\end{array}\right.
\end{aligned}
$$

Choose the actual controller as

$$
\begin{aligned}
u & =\alpha_{1}+\tau_{b}\left(c_{0} c_{1}+G^{-1} c_{1}^{2}\right) z_{1}-\frac{\tau_{b} c_{0}\left(c_{0} G+c_{1}\right)+\tau_{b} G-c_{0}}{c_{0}} z_{2} \\
& +\tau_{b} c_{1} G^{-1} z_{1} \frac{\partial f\left(x_{1}\right)}{\partial x_{1}}-\tau_{b} z_{2} \frac{\partial f\left(x_{1}\right)}{\partial x_{1}} \\
& -\tau_{b}\left(c_{1}+\frac{\partial f\left(x_{1}\right)}{\partial x_{1}}\right)^{2} \frac{\sigma}{G^{2} \gamma^{2}}-h_{1} \sigma-h_{2} \operatorname{sgn}(\sigma),
\end{aligned}
$$

where $h_{1}$ and $h_{2}$ are positive constants, and $\operatorname{sgn}(\sigma)$ denotes signum function.

Substituting the actual controller expressed by Eq. (27) into Eq. (26), we can obtain

$$
\begin{aligned}
& H_{2}=-c_{1} z_{1}^{2}+G z_{1} z_{2}+z_{1} d_{1}+\frac{1}{2}\left(\kappa_{1}^{2} z_{1}^{2}+\kappa_{2}^{2} z_{2}^{2}-\gamma^{2} d_{1}^{2}-\gamma^{2} d_{2}^{2}\right) \\
& +\sigma\left(\begin{array}{l}
-\frac{G}{c_{0}} z_{2}+c_{0} d_{1}+d_{2}+G^{-1}\left(c_{1}+\frac{\partial f\left(x_{1}\right)}{\partial x_{1}}\right) d_{1} \\
-\left(c_{1}+\frac{\partial f\left(x_{1}\right)}{\partial x_{1}}\right)^{2} \frac{\sigma}{G^{2} \gamma^{2}}-\frac{h_{1}}{\tau_{b}} \sigma-\frac{h_{2}}{\tau_{b}} \operatorname{sgn}(\sigma)
\end{array}\right) \\
& =-c_{1} z_{1}^{2}-\frac{G}{c_{0}} z_{2}^{2}+\left(c_{0}^{2}+1\right) z_{1} d_{1}+c_{0} z_{1} d_{2}+c_{0} z_{2} d_{1} \\
& +z_{2} d_{2}+\frac{1}{2}\left(\kappa_{1}^{2} z_{1}^{2}+\kappa_{2}^{2} z_{2}^{2}-\gamma^{2} d_{1}^{2}-\gamma^{2} d_{2}^{2}\right)+\frac{\gamma^{2}}{4} d_{1}^{2} \\
& -\left(\frac{\gamma}{2} d_{1}-\left(c_{1}+\frac{\partial f\left(x_{1}\right)}{\partial x_{1}}\right) \frac{\sigma}{G \gamma}\right)^{2}-\frac{h_{1}}{\tau_{b}} \sigma^{2}-\frac{h_{2}}{\tau_{b}} \sigma \operatorname{sgn}(\sigma) \text {. }
\end{aligned}
$$

$$
\begin{aligned}
& \left(c_{0}^{2}+1\right) z_{1} d_{1} \leq \frac{2\left(c_{0}^{2}+1\right)^{2}}{\gamma^{2}} z_{1}^{2}+\frac{1}{8} \gamma^{2} d_{1}^{2} \\
& c_{0} z_{1} d_{2} \leq \frac{c_{0}^{2}}{\gamma^{2}} z_{1}^{2}+\frac{1}{4} \gamma^{2} d_{2}^{2} \\
& c_{0} z_{2} d_{1} \leq \frac{2 c_{0}^{2}}{\gamma^{2}} z_{2}^{2}+\frac{1}{8} \gamma^{2} d_{1}^{2} \\
& z_{2} d_{2} \leq \frac{1}{\gamma^{2}} z_{2}^{2}+\frac{1}{4} \gamma^{2} d_{2}^{2} .
\end{aligned}
$$

Substituting the inequalities (29), (30), (31) and (32) into Eq. (28) yields

$$
\begin{aligned}
H_{2} \leq & -\left(c_{1}-\frac{2\left(c_{0}^{2}+1\right)^{2}}{\gamma^{2}}-\frac{c_{0}^{2}}{\gamma^{2}}-\frac{\kappa_{1}^{2}}{2}\right) z_{1}^{2} \\
& -\left(\frac{G}{c_{0}}-\frac{2 c_{0}^{2}}{\gamma^{2}}-\frac{1}{\gamma^{2}}-\frac{\kappa_{2}^{2}}{2}\right) z_{2}^{2} \\
& -\left(\frac{\gamma}{2} d_{1}-\left(c_{1}+\frac{\partial f\left(x_{1}\right)}{\partial x_{1}}\right) \frac{\sigma}{G \gamma}\right)^{2} \\
& -\frac{h_{1}}{\tau_{b}} \sigma^{2}-\frac{h_{2}}{\tau_{b}} \sigma \operatorname{sgn}(\sigma)
\end{aligned}
$$

Choose the parameters $c_{0}$ and $c_{1}$ that satisfying the following inequalities

$$
\frac{c_{1}-\frac{2\left(c_{0}^{2}+1\right)^{2}}{\gamma^{2}}-\frac{c_{0}^{2}}{\gamma^{2}}-\frac{\kappa_{1}^{2}}{2}>0}{\frac{G}{c_{0}}-\frac{2 c_{0}^{2}}{\gamma^{2}}-\frac{1}{\gamma^{2}}-\frac{\kappa_{2}^{2}}{2}>0 .}
$$

Substituting the inequalities (34) and (35) into inequality (33), we can obtain

$$
H_{2} \leq 0 \text {. }
$$

Defining $V(x)=V_{2}(x)$, and on the basis of Eq. (25) and inequality (36), we can obtain

$$
\dot{V} \leq \frac{1}{2}\left(\gamma^{2}\|d\|^{2}-\|z\|^{2}\right) .
$$


By the inequality (37), we can obtain the following inequality when the disturbance $d=0$

$$
\dot{V} \leq-\frac{1}{2}\|z\|^{2}
$$

Integrating both sides of the inequality (38), we can obtain

$$
\int_{0}^{\infty}\|z\|^{2} d t \leq 2(V(x(0))-V(x(\infty))) .
$$

Since $V(x(0))$ is bounded and $V(x(t))$ is a positive, non-increasing function, we can obtain $z \in L_{2}$. In addition, $z, \alpha_{1}, \dot{\alpha}_{1}, f\left(x_{1}\right), d_{1}, d_{2} \in L_{\infty}$, and we can get $\dot{z} \in L_{\infty}$ using Eqs. (15) and (20). With $z \in L_{2} \cap L_{\infty}$ and $\dot{z} \in L_{\infty}$, we can get $\lim _{t \rightarrow 0} z(t)=0$ based on Barbalat's lemma [26]. That implies that the closed-loop wheel dynamic system is asymptotically stable when the disturbance $d=0$.

Integrating both sides of the inequality (3r), we can obtain the following dissipative inequality when the disturbance $d \neq 0$

$$
V(x(T))-V(x(0)) \leq \frac{1}{2} \int_{0}^{\mathrm{T}}\left(\gamma^{2}\|d\|^{2}-\|z\|^{2}\right) d t .
$$

Therefore, the closed-loop wheel dynamic system with respect to the supply rate $w(d, z)=\frac{1}{2}\left(\gamma^{2}\|d\|^{2}-\|z\|^{2}\right)$ is dissipative, and according to the relationship between the dissipativity of the system and the $L_{2}$-gain stability of the system [22], the closed-loop wheel dynamic system is also $L_{2}$-gain stable.

Remark 1: It is easy to choose the parameters $c_{0}$ and $c_{1}$ to satisfy the inequalities (34) and (35), due to the relationship of the parameters $c_{0}$ and $c_{1}$ is decoupled.

Remark 2: To eliminate the chattering phenomenon due to the actual control input containing the discontinuous signum function, the subsequent continuous saturation function is employed to replace the discontinuous term [11]

$$
\operatorname{sat}(\sigma, \varepsilon)=\left\{\begin{array}{ll}
\frac{\sigma}{\varepsilon} & \text { if }|\sigma|<\varepsilon \\
\operatorname{sgn}(\sigma) & \text { if }|\sigma| \geq \varepsilon
\end{array},\right.
$$

where $\varepsilon>0$ is the width of the boundary layer. Thus, the actual controller in Eq. (27) is rewritten

$$
\begin{aligned}
u= & \alpha_{1}+\tau_{b}\left(c_{0} c_{1}+G^{-1} c_{1}^{2}\right) z_{1} \\
& -\frac{\tau_{b} c_{0}\left(c_{0} G+c_{1}\right)+\tau_{b} G-c_{0}}{c_{0}} z_{2} \\
& +\tau_{b} c_{1} G^{-1} z_{1} \frac{\partial f\left(x_{1}\right)}{\partial x_{1}}-\tau_{b} z_{2} \frac{\partial f\left(x_{1}\right)}{\partial x_{1}} \\
& -\tau_{b}\left(c_{1}+\frac{\partial f\left(x_{1}\right)}{\partial x_{1}}\right)^{2} \frac{\sigma}{G^{2} \gamma^{2}}-h_{1} \sigma-h_{2} \operatorname{sat}(\sigma, \varepsilon) .
\end{aligned}
$$

\section{Simulation Results}

The performance of the proposed RBSMC for reference input wheel slip tracking has been verified by a full-vehicle dynamics simulation model (MSC CarSim ${ }^{\circledR}$ ) with the actuator dynamics. MSC CarSim ${ }^{\circledR}$ is a comprehensive model for the efficient simulation of the whole vehicle dynamics, and it includes powertrain model, suspension model, aerodynamic model, and tire model with dynamic rolling resistance and relaxation length, etc. Therefore, the following simulation results can be considered very close to real-vehicle experiments. Straight line braking manoeuvres on a flat dry asphalt $\operatorname{road}(\mu=1)$ and a flat wet asphalt road $(\mu=0.6)$ are performed for testing the performance of the proposed RBSMC. All parameters of the proposed RBSMC are set by $\kappa_{1}=10, \kappa_{2}=0.01, c_{0}=1, c_{1}=350, \gamma=50, h_{1}=3.2$, $h_{2}=6$ and $\varepsilon=1$. The main parameters of the full-vehicle dynamics simulation model are set by $m=354 \mathrm{~kg}$, $J=0.9 \mathrm{~kg} \cdot \mathrm{m}^{2}$ and $r=0.31 \mathrm{~m}$.

First, straight line braking manoeuvre on a flat dry asphalt road is implemented under the conditions that the initial vehicle speed is set to $27.78 \mathrm{~m} / \mathrm{s}$ (equivalently $100 \mathrm{~km} / \mathrm{h}$ ) and the reference input wheel slip is set to 0.1, 0.06, and 0.03, respectively, and Figures 2-4 show the simulation results for comparing the performance of the proposed RBSMC with those of the sliding mode controller (SMC) for reference input wheel slip tracking. As shown in Figure 2(a), Figure 3(a) and Figure 4(a), both RBSMC and SMC exit when the vehicle speed is less than $4 \mathrm{~m} / \mathrm{s}$ (equivalently $14.4 \mathrm{~km} / \mathrm{h}$ ), and the mechanism can avoid RBSMC and SMC singularity and satisfy the engineering demands. Meanwhile, the vehicle speeds and wheel speeds of RBSMC are smaller than those of SMC at the same time respectively. As shown in Figure 2(b), Figure 3(b) and Figure 4(b), the wheel slips 


\section{Figure 2}

The simulation results of straight line braking manoeuvre on dry asphalt road surface with $\lambda_{d}=0.1$ : (a) vehicle speed and wheel speed, (b) reference input wheel slip and actual wheel slip, (c) tire-road friction coefficient versus wheel slip, and (d) braking torque

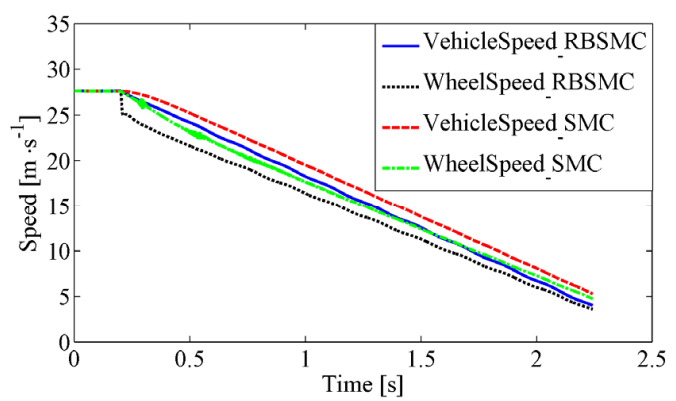

(a)

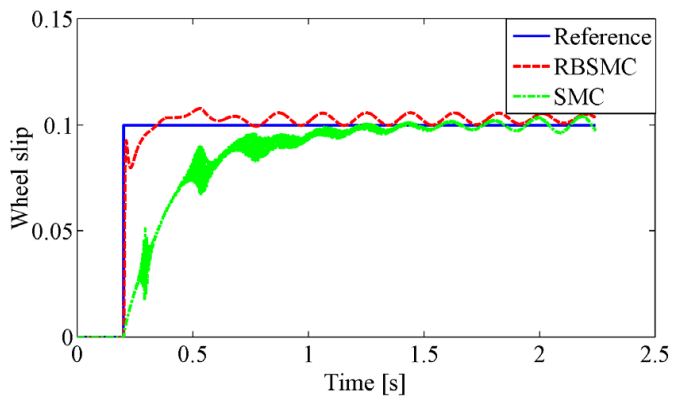

(b)

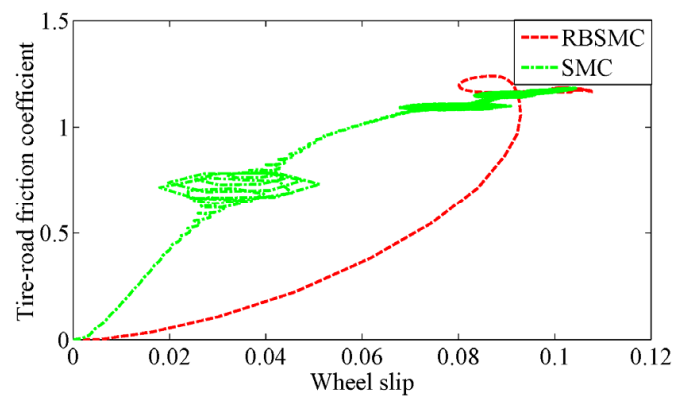

(c)

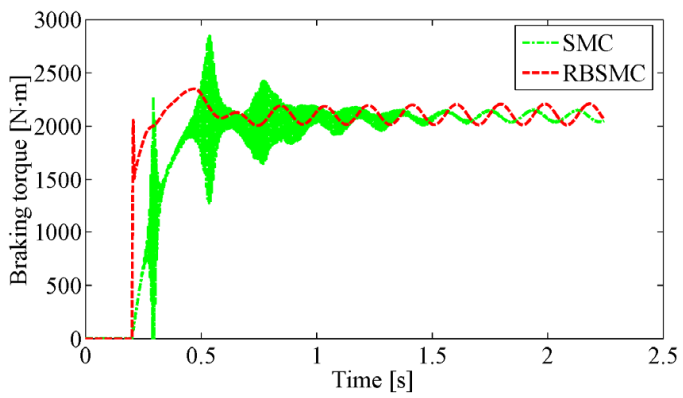

(d)
Figure 3

The simulation results of straight line braking manoeuvre on dry asphalt road surface with $\lambda_{d}=0.06$ : (a) vehicle speed and wheel speed, (b) reference input wheel slip and actual wheel slip, (c) tire-road friction coefficient versus wheel slip, and (d) braking torque

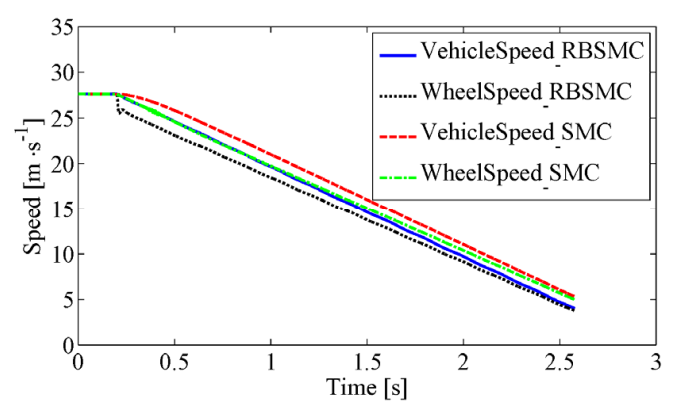

(a)

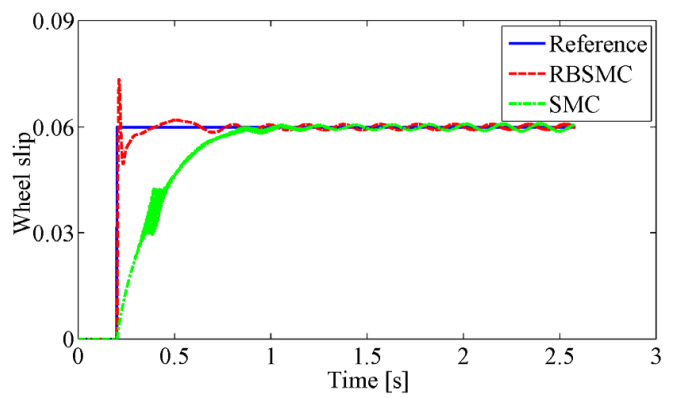

(b)

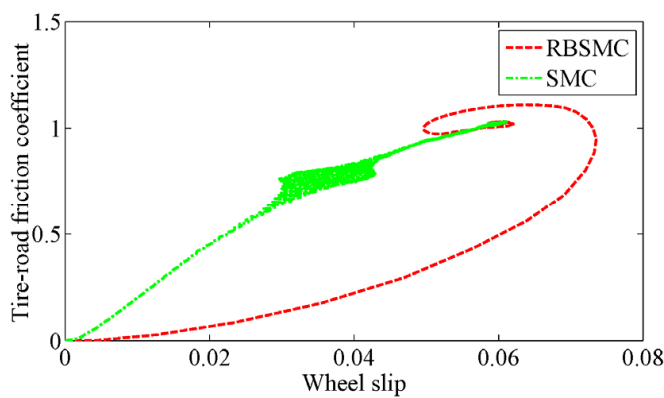

(c)

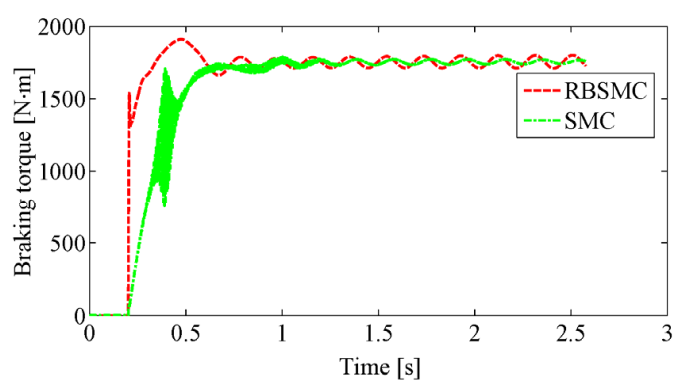

(d) 


\section{Figure 4}

The simulation results of straight line braking manoeuvre on dry asphalt road surface with $\lambda_{d}=0.03$ : (a) vehicle speed and wheel speed, (b) reference input wheel slip and actual wheel slip, (c) tire-road friction coefficient versus wheel slip, and (d) braking torque

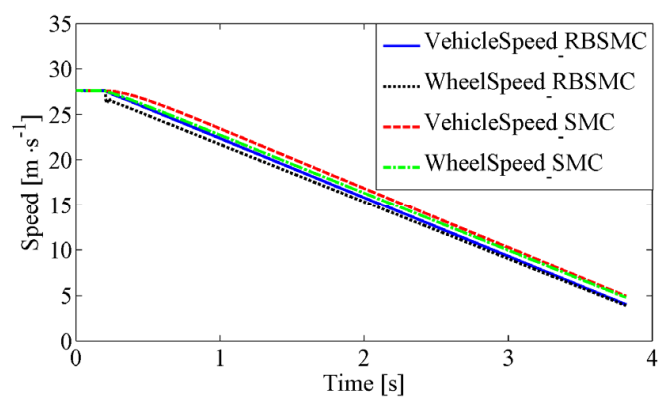

(a)

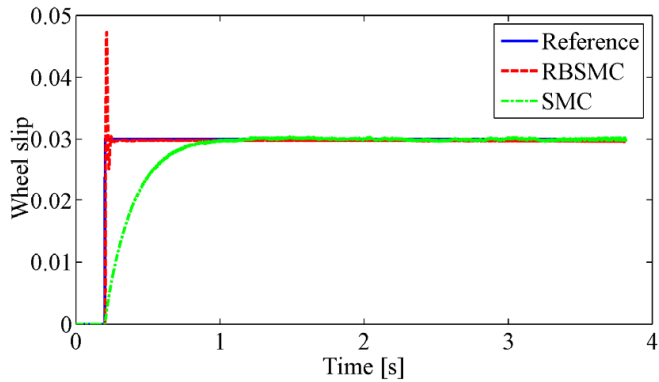

(b)

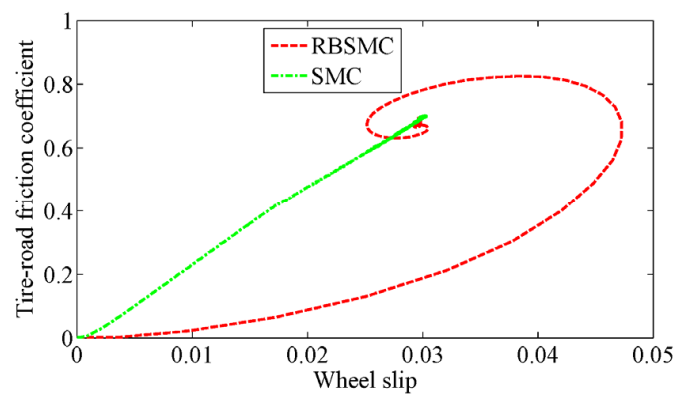

(c)

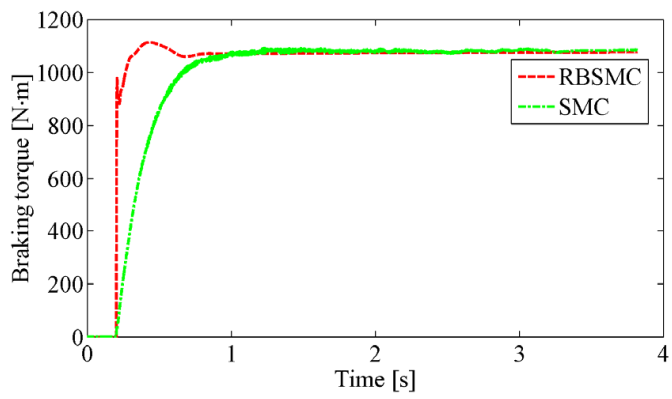

(d)
Figure 5

The simulation results of straight line braking manoeuvre on wet asphalt road surface with $\lambda_{d}=0.1$ : (a) vehicle speed and wheel speed, (b) reference input wheel slip and actual wheel slip, (c) tire-road friction coefficient versus wheel slip, and (d) braking torque

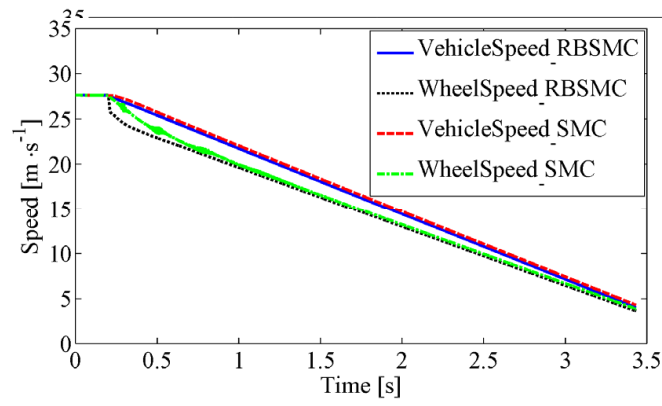

(a)

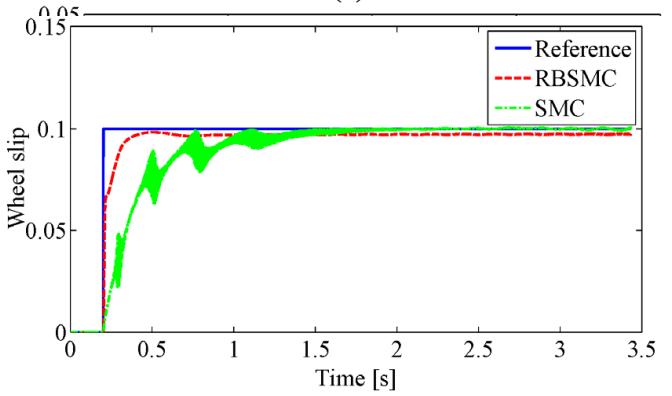

(b)

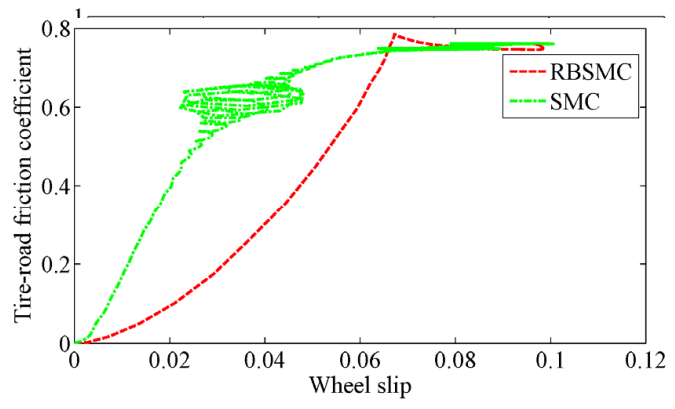

(c)

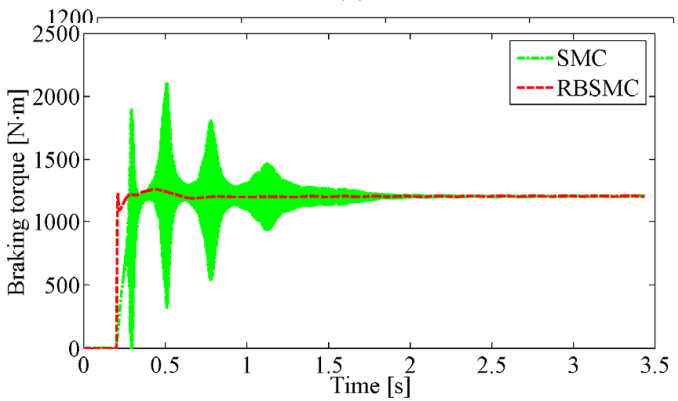

(d) 
Figure 6

The simulation results of straight line braking manoeuvre on wet asphalt road surface with $\lambda_{d}=0.06$ : (a) vehicle speed and wheel speed, (b) reference input wheel slip and actual wheel slip, (c) tire-road friction coefficient versus wheel slip, and (d) braking torque

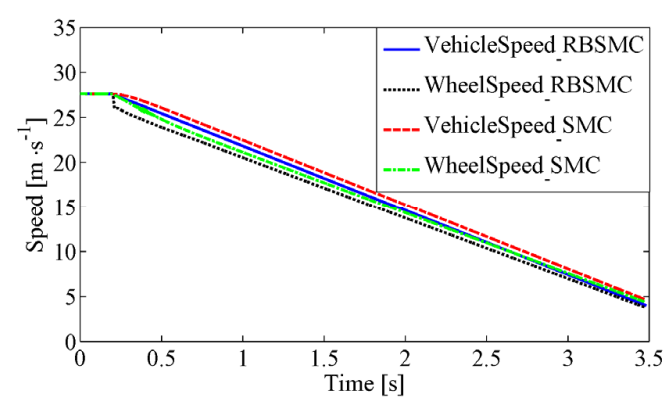

(a)

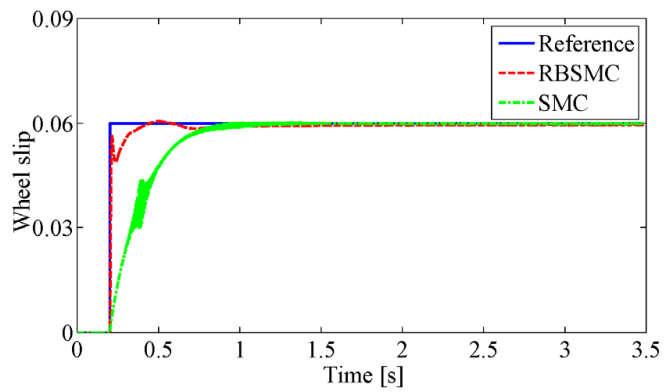

(b)

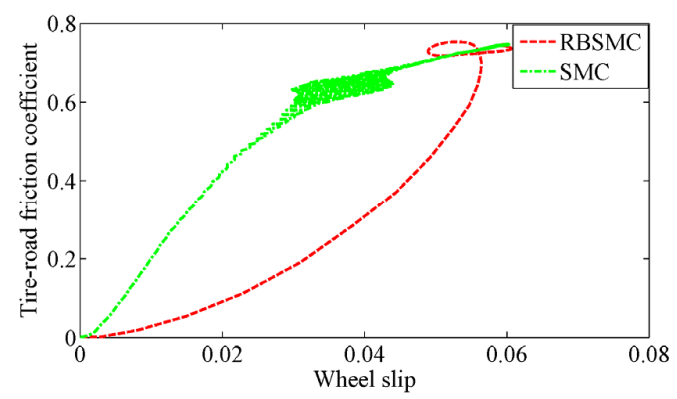

(c)

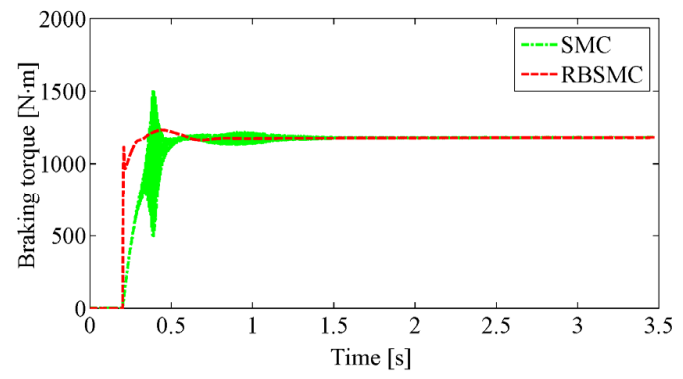

(d)
Figure 7

The simulation results of straight line braking manoeuvre on wet asphalt road surface with $\lambda_{d}=0.03$ : (a) vehicle speed and wheel speed, (b) reference input wheel slip and actual wheel slip, (c) tire-road friction coefficient versus wheel slip, and (d) braking torque

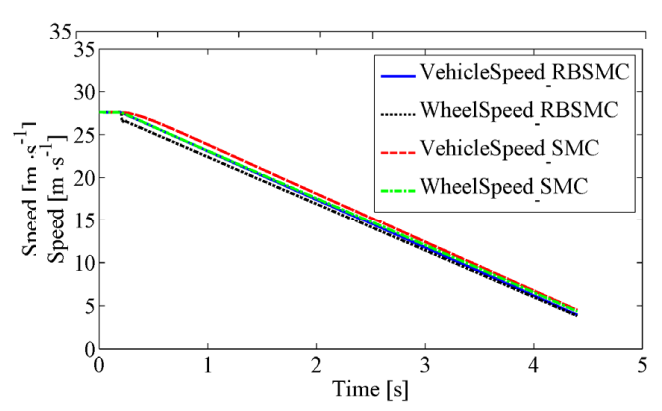

(a)

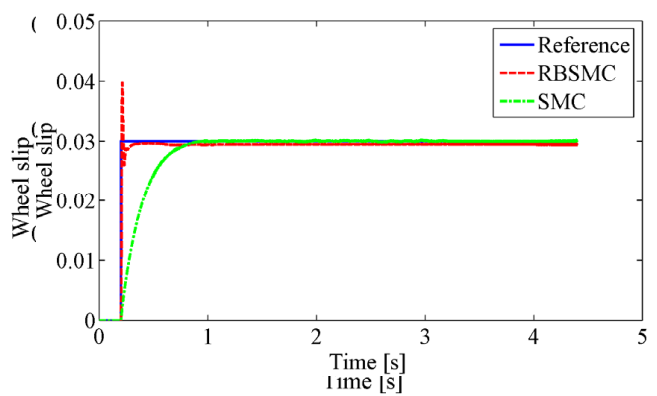

(b)

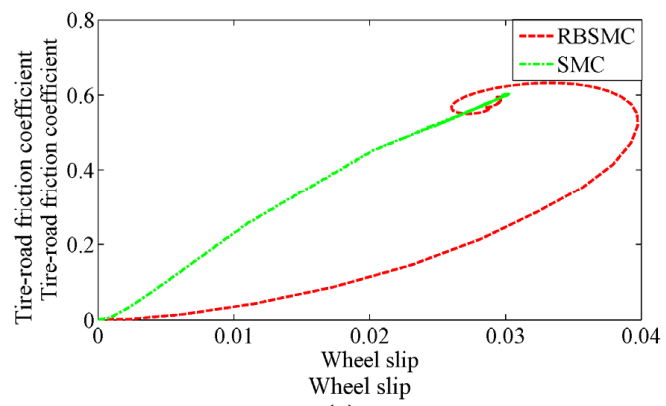

(c)

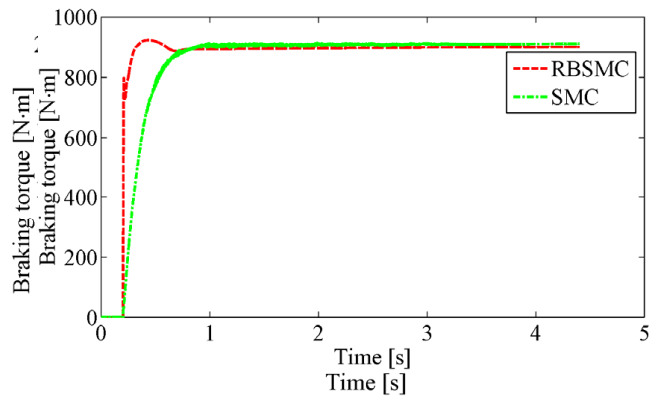

(d) 
of RBSMC have faster dynamic response and better tracking precision than those of SMC in the transient state. That explains why the vehicle speeds and wheel speeds of RBSMC are smaller than those of SMC at the same time respectively. Figure 2(c), Figure 3(c) and Figure 4(c) show that the wheel slips of RBSMC have similar performance with those of SMC in the steady state. As shown in Figure 2(d), Figure 3(d) and Figure 4(d), the braking torques of RBSMC are more smoother than those of SMC. Moreover, in order to quantitatively evaluate the performance of the RBSMC, the root mean-squared error (RMSE) between the reference input wheel slip and the actual value is computed. According to the statistical results shown as Table 2, the maximum RMSE of the proposed RBSMC in the straight line braking manoeuvre on a flat dry asphalt road is 0.0059, while the maximum RMSE of SMC is 0.0219 .

\section{Table 2}

Root mean-square error between the reference input wheel slip and actual value

\begin{tabular}{l|c|c|c}
\multirow{2}{*}{ Manoeuvre } & \multirow{2}{*}{ Reference } & \multicolumn{2}{|c}{ RMSE } \\
\cline { 2 - 4 } & 0.1 & RBSMC & SMC \\
\hline \multirow{2}{*}{$\begin{array}{l}\text { Straight line } \\
\text { braking on dry } \\
\text { asphalt road }\end{array}$} & 0.06 & 0.0025 & 0.0219 \\
\cline { 2 - 4 } & 0.03 & 0.0011 & 0.0118 \\
\hline $\begin{array}{l}\text { Straight line } \\
\text { braking on wet } \\
\text { asphalt road }\end{array}$ & 0.1 & 0.0064 & 0.0176 \\
\cline { 2 - 4 } & 0.06 & 0.0025 & 0.0099 \\
\hline
\end{tabular}

Second, straight line braking manoeuvre on a flat wet asphalt road is implemented with setting the initial vehicle speed $27.78 \mathrm{~m} / \mathrm{s}$ (equivalently $100 \mathrm{~km} / \mathrm{h}$ ) and the desired wheel slip 0.1, 0.06, and 0.03, respectively, and Figures 5-7 show the simulation results for comparing the performance of the proposed RBSMC with those of SMC. The similar results can be obtained

\section{References}

1. Alzer, H., Fonseca, C. M. D., Kovacec, A. Young-type Inequalities and Their Matrix Analogues. Linear and Multilinear Algebra, 2015, 63(3), 622-635. https://doi. org/10.1080/03081087.2014.891588 compared with straight line braking manoeuvre on a flat dry asphalt road. Both RBSMC and SMC have great robustness against the system uncertainty and external disturbance, but the proposed RBSMC has better performance in the transient state. According to the statistical results shown as Table 2, the maximum RMSE of the proposed RBSMC in the straight line braking manoeuvre on a flat wet asphalt road is 0.0064 , while the maximum RMSE of SMC is 0.0176.

\section{Simulation Results}

This paper has presented a new robust backstepping sliding mode controller (RBSMC) for reference input wheel slip tracking based on the single-corner model with the actuator dynamics. The proposed RBSMC combines the merit of backstepping method that has simplified and flexible design procedure with the merit of sliding mode control that is insensitive to system uncertainty and external disturbance, and the Lyapunov-based method is used to derive that the closedloop wheel dynamic system is $L_{2}$-gain stable. Then, the simulation based on the full-vehicle dynamics simulation model is implemented to validate the performance of the proposed RBSMC. The simulation results indicate that it can guarantee that the wheel slip follow the trend of the reference input quickly and accurately compared with the traditional sliding mode controller for reference input wheel slip tracking.

In future works, the proposed RBSMC will be further tested and fine tuned on a real test vehicle equipped with by-wire electro-mechanical-brakes. Moreover, active safety control systems and intelligent driver assistance systems based on the proposed RBSMC should be researched.

\section{Acknowledgement}

This work is supported by National Key Research and Development Program of China (Grant No. 2016YFB0101002).

2. Amodeo, M., Ferrara,A., Terzaghi, R., Vecchio, C. Wheel Slip Control via Second-Order Sliding-Mode Generation. IEEE Transactions on Intelligent Transportation Systems, 2010, 11(1), 122-131. https://doi.org/10.1109/TITS.2009.2035438 
3. Burckhardt, M. Fahrwerktechnik: Radschlupf-Regelsysteme. Vogel Verlag, Würzburg, 1993.

4. Choi, S. B. Antilock Brake System with a Continuous Wheel Slip Control to Maximize the Braking Performance and the Ride Quality. IEEE Transactions on Control Systems Technology, 2008, 16(5), 996-1003. https://doi.org/10.1109/TCST.2007.916308

5. Das, A., Lewis, F., Subbarao, K. Backstepping Approach for Controlling a Quadrotor Using Lagrange Form Dynamics. Journal of Intelligent \& Robotic Systems, 2009, 56(1), 127-151. https://doi.org/10.1007/s10846-0099331-0

6. Harifi, A., Aghagolzadeh, A., Alizadeh, G., Sadeghi, M. Designing a Sliding Mode Controller for Slip Control of Antilock Brake Systems. Transportation Research Part C, 2008, 16, 731-741. https://doi.org/10.1016/j. trc.2008.02.003

7. Hsu, C. F. Intelligent Exponential Sliding-Mode Control with Uncertainty Estimator for Antilock Braking Systems. Neural Computing and Applications, 2016, 27(6), 1463-1475. https://doi.org/10.1007/s00521-015-1946-4

8. Jing, H. H., Liu, Z. Y., Chen, H. A Switched Control Strategy for Antilock Braking System with on/off Valves. IEEE Transactions on Vehicular Technology, 2011, 60(4), 1470-1484. https://doi.org/10.1109/ TVT.2011.2125806

9. Johansen, T. A., Petersen, I., Kalkkuhl, J., Lüdemann, J. Gain-Scheduled Wheel Slip Control in Automotive Brake Systems. IEEE Transactions on Control Systems Technology, 2003, 11(6), 799-811. https://doi. org/10.1109/TCST.2003.815607

10. Kiencke, U., Nielsen, L. Automotive Control Systems. Springer, Berlin, 2000.

11. Lee, D. Spacecraft Coupled Tracking Maneuver Using Sliding Mode Control with Input Saturation. Journal of Aerospace Engineering, 2015, 28(5), 401-413. https:// doi.org/10.1061/(ASCE)AS.1943-5525.0000473

12. Lin, C. M., Hsu, C. F. Neural-Network Hybrid Control for Antilock Braking Systems. IEEE Transactions on Neural Networks, 2003, 14(2), 351-359. https://doi. org/10.1109/TNN.2002.806950

13. Mirzaei, A., Moallem, M., Dehkordi, B. M., Fahimi, B. Design of an Optimal Fuzzy Controller for Antilock Braking Systems. IEEE Transactions on Vehicular Technology, 2006, 55(6), 1725-1730. https://doi.org/10.1109/ TVT.2006.878714

14. Mirzaei, M., Mirzaeinejad, H. Optimal Design of a Non-linear Controller for Anti-lock Braking Sys- tem. Transportation Research Part C, 2012, 24, 19-35. https://doi.org/10.1016/j.trc.2012.01.008

15. Mirzaeinejad, H. Robust Predictive Control of Wheel Slip in Antilock Braking Systems Based on Radial Basis Function Neural Network. Applied Soft Computing, 2018, 70, 318-329. https://doi.org/10.1016/j. asoc.2018.05.043

16. Moon, S., Moon, I., Yi, K. Design, Tuning, and Evaluation of a Full-range Adaptive Cruise Control System with Collision Avoidance. Control Engineering Practice, 2009, 17(4), 442-455. https://doi.org/10.1016/j.conengprac.2008.09.006

17. Park, J. H. Synchronization of Genesio Chaotic System via Backstepping Approach. Chaos, Solitons \& Fractals, 2006, 27(5), 1369-1375. https://doi.org/10.1016/j.chaos.2005.05.001

18. Park, K. S., Lim, J. T. Wheel Slip Control for ABS with Time Delay Input using Feedback Linearization and Adaptive Sliding Mode Control. International Conference on Control, Automation and Systems, Seoul, Korea, 2008, 290-295. https://doi.org/10.1109/ICCAS.2008.4694658

19. Pasillas-Lépine, W. Hybrid Modelling and Limit Cycle Analysis for a Class of Five-phase ABS Algorithms. Vehicle System Dynamics, 2006, 44(2), 173-188. https:// doi.org/10.1080/00423110500385873

20. Sardarmehni, T., Rahmani, H., Menhaj, M. B. Robust Control of Wheel Slip in Anti-lock Brake System of Automobiles. Nonlinear Dynamics, 2014, 76(1), 125-138. https://doi.org/10.1007/s11071-013-1115-1

21. Savaresi, S. M., Tanelli, M. Active Braking Control Systems Design for Vehicles. Springer, London, 2010. https://doi.org/10.1007/978-1-84996-350-3

22. Schaft, A. V. D. L2-gain and Passivity Techniques in Nonlinear Control. Springer, Berlin, 1996. https://doi. org/10.1007/3-540-76074-1

23. Shim, T., Chang, S., Lee, S. Investigation of Sliding-Surface Design on the Performance of Sliding Mode Controller in Antilock Braking Systems. IEEE Transactions on Vehicular Technology, 2008, 57(2), 747-759. https:// doi.org/10.1109/TVT.2007.905391

24. Tanelli, M., Astolfi, A., Savaresi, S. M. Robust Nonlinear Output Feedback Control for Brake by Wire Control Systems. Automatica, 2008, 44, 1078-1087. https://doi. org/10.1016/j.automatica.2007.08.020

25. Tanelli, M., Osorio, G., Bernardo, M. D., Savaresi, S. M., Astolfi, A. Existence, Stability and Robustness Analysis of Limit Cycles in Hybrid Anti-lock Braking Systems. 
International Journal of Vehicle Design, 2009, 82(4), 659-678. https://doi.org/10.1080/00207170802203598

26. Tao, G. A Simple Alternative to the Barbalat Lemma. IEEE Transations on Automatic Control, 1997, 42(5), 698. https://doi.org/10.1109/9.580878

27. Yu, H. X., Qi, Z. Q., Duan, J. M., Taheri, S., Ma, Y. F. Multiple Model Adaptive Backstepping Control for Antilock Braking System based on LuGre Dynamic Tyre Model. International Journal of Vehicle Design, 2015, 69(1/2/3/4), 168-184. https://doi.org/10.1504/ IJVD.2015.073120
28. Zhao, J., Hill, D. J. On Stability, L2-gain and $\mathrm{H} \infty$ Control for Switched Systems. Automatica, 2008, 44(5), 12201232.

29. Zhou, H. L., Liu, Z. Y. Vehicle Yaw Stability-Control System Design based on Sliding Mode and Backstepping Control Approach. IEEE Transactions on Vehicular Technology, 2010, 59(7), 3674-3678. https://doi. org/10.1109/TVT.2010.2050790

30. Zhu, Q., Zhang, T. P., Fei, S. M. Adaptive Tracking Control for Input Delayed MIMO Nonlinear Systems. Neurocomputing, 2012, 74, 472-480. https://doi.org/10.1016/j.neucom.2010.03.003 